\title{
Exciton-polariton topological insulator
}

\author{
S. Klembt ${ }^{1,+,}$, T.H. Harder ${ }^{1,+}$, O.A. Egorov ${ }^{1}$, K. Winkler ${ }^{1}$, R. Ge ${ }^{2}$, M.A. Bandres ${ }^{3}$, M. \\ Emmerling $^{1}$, L. Worschech ${ }^{1}$, T.C.H. Liew ${ }^{2}$, M. Segev ${ }^{3}$, C. Schneider ${ }^{1}$ and S. Höfling ${ }^{1,4}$ \\ ${ }^{1}$ Technische Physik and Wilhelm-Conrad-Röntgen-Research Center for Complex Material Systems, \\ Universität Würzburg, Am Hubland, 97074 Würzburg, Germany \\ ${ }^{2}$ Division of Physics and Applied Physics, School of Physical and Mathematical Sciences, Nanyang \\ Technological University, 21 Nanyang Link, 637371, Singapore \\ ${ }^{3}$ Physics Department and Solid State Institute, Technion, Haifa 32000, Israel \\ ${ }^{4}$ SUPA, School of Physics and Astronomy, University of St. Andrews, KY 16 9SS, United Kingdom \\ +These authors contributed equally to this work \\ *sebastian.klembt@physik.uni-wuerzburg.de
}

Topological insulators are a striking example of materials in which topological invariants are manifested in robustness against perturbations [1]. Their most prominent feature is the emergence of topological edge states with reduced dimension at the boundary between areas with distinct topological invariants. The observable physical effect is unidirectional robust transport, unaffected by defects or disorder. Topological insulators were originally observed in the integer quantum Hall effect [2], and subsequently suggested [3-5] and observed [6] even in the absence of magnetic field. These were fermionic systems of correlated electrons. However, during the past decade the concepts of topological physics have been introduced into numerous fields beyond condensed matter, ranging from microwaves $[7,8]$ and photonic systems [9,10] to cold atoms [11], acoustics [12,13] and even mechanics [14]. Recently, topological insulators were proposed in exciton-polariton systems [15-17] organized as honeycomb (graphene-like) lattices, under the influence of a magnetic field. Topological phenomena in exciton-polaritons are fundamentally different from all topological effects demonstrated experimentally thus far: exciton-polaritons are part-light part-matter 
quasiparticles emerging from the strong coupling of quantum well excitons and cavity photons [18]. Here, we demonstrate experimentally the first exciton-polariton topological insulator. This constitutes the first symbiotic light-matter topological insulators. Our polariton lattice is excited non-resonantly, and the chiral topological polariton edge mode is populated by a polariton condensation mechanism. We use scanning imaging techniques in real-space and in Fourier-space to measure photoluminescence, and demonstrate that the topological edge mode avoids defects, and that the propagation direction of the mode can be reversed by inverting the applied magnetic field. Our exciton-polariton topological insulator paves the way for a variety of new topological phenomena, as they involve light-matter interaction, gain, and perhaps most importantly - exciton-polaritons interact with one another as a nonlinear many-body system.

Microcavity exciton-polaritons (polaritons) are composite bosons originating from the strong coupling of quantum well excitons to microcavity photons. While the excitonic fraction provides a strong non-linearity, the photonic part results in a low effective mass, allowing the formation of a driven-dissipative Bose-Einstein condensate [19], making polaritons being referred to as „quantum fluids of light“ [20]. For the epitaxially well-controlled III-V semiconductor material system, a variety of techniques are available to micropattern such cavities in order to precisely engineer the potential landscapes of polaritons [21]. With the recent advances of bringing topological effects to the realms of photonics and polaritonics [7-10,22], several avenues to realize topological edge propagation with polaritons have been suggested [15-17], with honeycomb geometries ("artificial graphene” [23]) being of particular interest to realize a $C=2$ Chern band insulator [17]. Indeed, polariton honeycomb lattices have been found to support Dirac-cone dispersions [24] as well as edge modes [25] inherited from their graphene origin [26,27]. Here, we 
take the next step and realize a topological Chern insulator in the first symbiotic part-light part matter system: the system of exciton-polaritons. Our experiments are based on the proposals [1517] of a honeycomb potential landscape for exciton-polaritons with its time-reversal symmetry broken by an applied magnetic field.

Let us first introduce the underlying physics and show its features in numerical simulations with a realistic set of sample parameters. The experiments display reliable condensation of polaritons at the vicinity of the Dirac cones and the creation of topological edge modes enabled by applying a magnetic field. Hence, we consider the injection of polaritons into the topological gap and focus on the inherent properties of the chiral edge modes.

A general schematic of the experiment is presented in Fig. 1a, while Figs. 1b and 1c depict the calculated dispersion relation of the honeycomb structure in the direction $\Gamma \rightarrow K$, connecting two Dirac cones ( $K$ and $K^{\prime}$ ), without and with applied magnetic field, respectively. The effective spinorbit coupling of polaritons, induced by TE-TM mode splitting, breaks the polarization-related symmetry and thus each Dirac point transforms into four inverted parabolas [17]. While the spinorbit interaction is extremely small in real graphene [28], "polariton graphene" offers the possibility to make the effective spit-orbit coupling sufficiently large to open a sizeable gap in a magnetic field. Without a magnetic field, the two central parabolas touch each other at the Dirac cones of the underlying honeycomb lattice (Fig. 1b). The degeneracy between the states in the crossing points can be lifted in the presence of a magnetic field and a finite Zeeman splitting. As a consequence, an energy gap forms in the vicinity of the Dirac cones (Fig. 1c). It is worth mentioning that the Dirac cones $K$ and $K^{\prime}$ are not equivalent. At the $K\left(K^{\prime}\right)$ point, the "valence" band is formed from the $B(A)$ pillars and the "conduction" band is formed from the $A(B)$ pillars 
[17]. The reversed order of the bands in the basis of the sublattices signifies that the gap is topologically non-trivial [1].

The interplay of an external magnetic field and the effective spin-orbit coupling (TE-TM mode splitting) results in non-zero Berry connections around the $K$ and $K^{\prime}$ points, contributing to the total band Chern number $C= \pm 2[16,17]$. As a consequence, the honeycomb structure supports one-way propagating edge states for the energies within the topological gap. Fig. 1c demonstrates the results of a band structure calculation combined with the dispersion of the edge states localized at the zigzag edge of the honeycomb structure. The propagation direction of these edge states is related to the direction of the external magnetic field: the polariton edge current is either left moving (L, yellow) or right moving (R, red), depicted in Fig. 1a, depending on the sign of the magnetic field. Fig. 1e depicts the corresponding calculated edge mode.

To illustrate the existence and robustness of the topologically-protected one-way edge states, we simulate the evolution of a wavepacket excited locally (Figs. 1f, g (red circle)) at the zigzag edge of the honeycomb structure. Figs. $1 \mathrm{f}$ and $1 \mathrm{~g}$ show that the launched wave packet starts to propagate left or right along the edge, depending on the polarity of the magnetic field, whereas in the absence of magnetic field, the launched wave packet remains at the excitation point. Note that the overall intensity decreases with propagation, since the model takes into account a realistic polariton lifetime of $\sim 35$ ps (see Methods for details). Furthermore, the chiral edge mode is topologically protected since it propagates along a $90^{\circ}$ corner and is able to bypass a point-like defect (see Methods).

Having established the features we expect to observe in an exciton-polariton topological insulator, we describe the experimental platform. To realize a polariton honeycomb potential lattice, we fabricate a planar microcavity containing three $\operatorname{In}_{0.04} \mathrm{Ga}_{0.96} \mathrm{As}$ quantum wells (QWs) in 
a $\lambda$-cavity, sandwiched between two GaAs/AlGaAs distributed Bragg reflectors (DBRs) with 30 (35.5) top (bottom) mirror pairs (Fig. 2a). Subsequently, we use electron beam lithography to define the honeycomb lattice, employing micropillars with a diameter of $d=2.0 \mu \mathrm{m}$ and a pillar-topillar overlap of $v=a / d=0.85$, where $a$ denotes the center-to-center distance between neighboring pillars. Finally, the upper DBR is etched in such a way that only two DBR pairs of the top DBR remain, so as not to damage the active QW region (Figs. 2b, c) (see Methods). When these sites are arranged in a two-dimensional lattice, the discrete pillar modes hybridize due to their proximity to one another and form a polaritonic band structure. The honeycomb lattice is characterized by a two-element base in real-space (Fig. 2d) and six degenerate $K$ - and $K^{\prime}$-points supporting Dirac cones in the first Brillouin zone (Fig. 2e), as well known from graphene. Fig. $2 \mathrm{f}$ depicts characterization of the polariton honeycomb lattice in the linear regime using non-resonant laser excitation. The Fourier-space energy-resolved photoluminescence (PL) of the investigated lattice is imaged in $k_{\mathrm{y}}$-direction, and scanned in $k_{\mathrm{x}}$-direction. The blue data points are fitted to the measured dispersions, accurately revealing the six Dirac cones at the $K$ - and $K^{\prime}$-points. The results of the corresponding tight-binding model (see Methods for details) are plotted in red and yellow, agreeing very well with the experimental data.

Next, we describe the experiments, conducted on the honeycomb exciton-polariton lattice under an external magnetic field, aiming to find topologically non-trivial edge states. To be able to observe a band gap that opens at the Dirac points, the Zeeman-splitting as well as the TE-TM splitting at the Dirac points, need to be sufficiently large compared to the PL linewidth. This implies that the polaritons need to have a sufficient excitonic part. Therefore, we select a lattice at a moderate negative detuning of $\delta=-11.5 \mathrm{meV}$ as summarized in the Methods Section. 
In order to assess the size of the bandgap, we apply polarization resolved spectroscopy, making use of a $\lambda / 4$ polarization series at an external magnetic field of $B=0 \mathrm{~T}$ and $5 \mathrm{~T}$. At an external magnetic field of $B=+5 \mathrm{~T}$, the Hopfield coefficients at the Dirac points return a photonic fraction of $|C|^{2}=0.96$ and an excitonic fraction of $|X|^{2}=0.04$. Furthermore, the TE-TM splitting at the wavevector of the Dirac point (i.e. $\left|k_{\triangleright}\right| \approx 0.77 \pi / \mathrm{a}$ ) can be estimated to be $400 \mu \mathrm{eV}$ for the photons, resulting in an effective TE-TM splitting for the exciton-polarions $\simeq 384 \mu \mathrm{eV}\left(\beta_{\mathrm{eff}} \simeq 263 \mu \mathrm{eV} \mu \mathrm{m}^{2}\right)$. The Zeeman-splitting of the excitonic mode is determined to be $\sim 540 \mu \mathrm{eV}$, leading to $\Delta_{\mathrm{eff}} \simeq 22 \mu \mathrm{eV}$ at the Dirac point (see Methods). As the TE-TM-splitting is considerably larger than the Zeemansplitting in the lattice studied here, the experimentally determined band gap that opens due to the magnetic field is of $\Delta_{\mathrm{g}}=(108 \pm 32) \mu \mathrm{eV}$ with $\Delta_{\mathrm{eff}}<\Delta_{\mathrm{g}}<\beta_{\mathrm{eff}}$, which, as we show later on, is reasonable for observing the topological features of our lattice and compares well with a gap size of about $100 \mu \mathrm{eV}$ found for realistic system parameters in [16]. After having established that a band gap opens at the Dirac points under the influence of an external magnetic field, we employ nonresonant excitation, under conditions allowing for polariton condensation into a chiral edge state within this band gap. Scans of the PL signal in real space as well as Fourier space are performed on the polariton lattice at external magnetic fields of $B=-5 \mathrm{~T}, 0 \mathrm{~T}$ and $+5 \mathrm{~T}$. The sample is excited on the zigzag-edge by a pulsed and chopped large laser beam with a diameter of $40 \mu \mathrm{m}$ at $792 \mathrm{~nm}$ wavelength, tuned to the first stopband minimum (see Fig. 1a). In Fig. 3a the linear polariton dispersion in $K^{\prime} \rightarrow \Gamma \rightarrow K$ direction at $B=0 \mathrm{~T}$ and (b) at $B=+5 \mathrm{~T}$ is displayed. The Dirac-cone dispersion is clearly visible $(P \sim 0.1 \mathrm{~mW})$. At a threshold power of $P_{\text {thr }}=1.8 \mathrm{~mW}$, we observe a nonlinear increase of the output power as well as a sudden decrease in linewidth (see Methods for details), establishing that a polariton condensate has formed at the $K, K^{\prime}$-points at around $k_{\mathrm{y}} \approx \pm 0.77 \pi / \mathrm{a}$, as displayed in Fig. 3c. We now perform mode tomography, scanning the real space 
$(x, y)$ landscape and measuring the energy $E_{\mathrm{PL}}$. Fig. $3 d$ shows the spectrum of a line perpendicular to the zigzag edge, taken at the position indicated by the solid white line in Fig. 3g. The dashed white line depicts the edge of the sample. Remarkably, besides a S-band condensate throughout the excited structure at $E_{\mathrm{S}}=1.4674 \mathrm{eV}$, we observe an edge mode: a region of high intensity residing only at the outermost row of lattice sites spatially and spectrally at an energy of $1.4678 \mathrm{eV}$ (white ellipse) - the expected topological band gap.

Fig. 3e shows the intensity pattern integrated over the energetic range of the trivial $S$-band mode centered at $1.4674 \mathrm{eV}$. Clearly, the condensate is relatively homogeneous over a large fraction of the lattice. The white overlaid lattice geometry and the microscopy image insets illustrate the position of the edge of the sample. Now, by changing the energy of the mode tomography to an energy $E_{\text {edge }}=1.4678 \mathrm{eV}$, within the topological gap (under a magnetic field of $+5 \mathrm{~T}$ ), the existence of edge states becomes unequivocal (at $x \approx 8 \mu \mathrm{m}$ in Fig. 3g). The PL at this energy originates predominantly from the outermost row of lattice sites with almost no emission detected from the bulk of the lattice. The mode is in excellent agreement with the Bloch mode calculations in Figs. 1e, f, g. In addition, the theoretical description within a Ginzburg-Landau-based model confirms that indeed polariton condensation into the edge mode occurs (see Methods for details). We now move on to study the robustness of the topological edge state. We do that by installing an artificial defect into the lattice at $y=15 \mu \mathrm{m}$ (see white circle in Figs. 3e-g). The defect is formed by leaving one of the sites on the zigzag edge of the honeycomb lattice unoccupied (see Methods). Normally, such a strong defect would cause scattering into the bulk, but here (see Figs. 3f, g) such scattering is suppressed indicating that the transport of the topological edge state is immune to such defects. In addition, we perform a mode tomography using non-resonant excitation with a large spot at the corner position of the sample. When plotting the energy of the topological edge mode 
$E_{\text {edge }}=1.4678 \mathrm{eV}$ in Fig. $3 f$, one clearly observes that the mode extends around the corner from the zigzag into the armchair configuration, without any sign of backscattering or bulk scattering. The measurements at $B=-5 \mathrm{~T}$ show very similar behavior, but the transport is in the opposite direction. Remarkably, when the magnetic field is absent $(B=0 \mathrm{~T})$, the edge mode vanishes completely (see Methods for details). The observation of the edge mode around the corner and especially its existence at the armchair edge, where topologically-trivial honeycomb lattices do not have edge modes $[9,26]$, prove unequivocally that the edge mode we observe is indeed topological and is endowed with topological protection.

To get further insight into the nature of these edge states, we analyze hyperspectral images $\left(\left(k_{\mathrm{x}}, k_{\mathrm{y}}\right)\right.$ vs. $\left.E_{\mathrm{PL}}\right)$ to identify the dominant propagation direction, with and without magnetic field. The results are displayed in Fig. 4. While in real-space the modes can be clearly separated in energy, the integration over Fourier-space results in the topological edge mode and the trivial bulk modes to overlay on top of one another. Figs. 4a, b depict the integrated intensities of the full $S$ band condensate (1.467-1.468 eV) including the energies associated with the edge state, for experiments at $B=+5 \mathrm{~T}$ and $-5 \mathrm{~T}$, respectively. To analyze the directionality of polariton transport, the maximum peak intensities at the two maxima at $k_{\mathrm{x}} \approx 0$ and $k_{\mathrm{y}} \approx \pm 0.77 \pi / \mathrm{a}$ are extracted, by identifying the central pixel of the peak and averaging the intensity of a region of $3 \times 3$ pixels centered around this position. The vertical axis of Fig. 4c shows the ratio of the luminescence travelling in one direction $\left(+k_{\mathrm{y}}\right)$ to the opposite direction $\left(-k_{\mathrm{y}}\right)$. Deviation of this quantity from unity is an essential characteristic of a chiral state and an opposite deviation should appear for opposite applied magnetic field. The corresponding intensity ratios are plotted in blue and show a clear directionality when an external magnetic field is applied. The transport changes its predominant direction along the edge when the direction of the magnetic field is inverted. This 
observation supports the interpretation of the edge-states being a result of a topologically nontrivial band gap, with the edge mode contributing to the chirality along the edge. Reversing the magnetic field reverses the slope of the dispersion curve of the topological edge mode, which is physically manifested in reversing the group velocity. On the other hand, we find no systematic directionality for the peaks at $k_{\mathrm{x}} \approx-0.77 \pi / \mathrm{a}$ in Figs. $4 \mathrm{a}$, b, which implies that these arise solely from the bulk condensate.

The experimental results depicted in Figs. 3 and 4 prove, unequivocally, the observation of an exciton-polariton topological Chern insulator. The application of a magnetic field on the honeycomb lattice opens up a topological bandgap with topological edge states supporting unidirectional transport whose propagation direction is determined by the field polarity. The lack of scattering from an artificial defect manifests the robustness of the topological edge mode. Furthermore, the observation of the edge mode extending around the corner and at the armchair termination without bulk scattering is a distinct feature of the topological edge mode. Our results lead the way to efficient light trapping and topologically protected propagation of coherent exciton-polariton condensates in a well-developed semiconductor platform, where also electrical driving can be envisaged [29]. We now aim at further exploring the topological lasing aspect of these experiments, by comparing topological edge mode lasing to lasing from a trivial edge mode in e.g. a Semenoff insulator. Such experiments would also link our exciton-polariton platform to the recently observed topological insulator laser [30,31]. Due to the interacting nature of polaritons, the in-depth study of collective bosonic effects in topological insulators can be envisaged. For example, the large nonlinearity displayed by this exciton-polariton topological system can support the observation of solitons, which were proposed [32,33] but have thus far never been observed in any system. Altogether, this work paves the way towards new topological 
polaritonic devices with unique properties and functionalities involving nonlinearity, gain, interactions and coherence.

\section{References}

1. Hasan, M. Z. \& Kane, C. L. Colloquium: Topological Insulators. Rev. Mod. Phys. 82, 3045-3067 (2010).

2. Klitzing, K. v., Dorda, G. \& Pepper, M. New Method for High-Accuracy Determination of the Fine-Structure Constant Based on Quantized Hall Resistance. Phys. Rev. Lett. 45, 494 (1980).

3. Haldane, F. D. M., Model for a quantum hall effect without landau levels: Condensed-matter realization of the "parity anomaly". Phys. Rev. Lett. 61, 2015-2018 (1988).

4. Kane, C. L. \& Mele, E. J. Quantum Spin Hall Effect in Graphene. Phys. Rev. Lett. 95, 226801 (2005).

5. Bernevig, B. A., Hughes, T. L. \& Zhang, S.-C. Quantum spin Hall effect and topological phase transition in HgTe quantum wells. Science 314, 1757-1761 (2006).

6. König, M., Wiedmann, S., Brune, C., Roth, A., Buhmann, H., Molenkamp, L. W., Qi, X.-L. \& Zhang, S.-C. Quantum spin hall insulator state in HgTe quantum wells. Science 318, 766-770 (2007).

7. Haldane, F. D. M. \& Raghu, S. Possible Realization of Directional Optical Waveguides in Photonic Crystals with Broken Time-Reversal Symmetry. Phys. Rev. Lett. 100, 013904 (2008).

8. Wang, Z., Chong, Y.D., Joannopoulos, J.D., Soljacić, M., Observation of unidirectional backscattering-immune topological electromagnetic states. Nature 461, 772-5 (2009).

9. Rechtsman, M. C., Zeuner, J. M., Plotnik, Y., Lumer, Y., Podolsky, D., Dreisow, F., Nolte, S., Segev, M. \& Szameit, A. Photonic Floquet topological insulators. Nature 496, 196-200 (2013).

10. Hafezi, M., Mittal, S., Fan, J., Migdall, A. \& Taylor, J. M. Imaging topological edge states in silicon photonics. Nat. Photon. 7, 1001-1005 (2013).

11. Jotzu, G. et al., Experimental realization of the topological Haldane modelwith ultracold fermions. Nature 515, 237-240 (2014).

12. Yang, Z. et al., Topological Acoustics. Phys. Rev. Lett. 114, 114301 (2015).

13. Fleury, R., Khanikaev, A. B., Alù, A., Floquet topological insulators for sound. Nat. Commun. 7, 11744 (2016).

14. Süsstrunk, R. \& Huber, S. D., Observation of phononic helical edge states in a mechanical topological insulator, Science 349, 47-50, (2015).

15. Karzig, T., Bardyn, C.-E., Lindner, N. H. \& Refael, G. Topological Polaritons Phys. Rev. X 5, 031001 (2015).

16. Bardyn, C.-E., Karzig, T., Refael, G. \& Liew, T. C. H. Topological polaritons and excitons in garden-variety systems Phys. Rev. B 91, 161413(R) (2015).

17. Nalitov, A. V., Solnyshkov, D. D. \& Malpuech, G. Polariton $\mathbb{Z}$ Topological Insulator. Phys. Rev. Lett. 114, 116401 (2015).

18. Weisbuch, C., Nishioka, M., Ishikawa, A. \& Arakawa, Y. Observation of the coupled excitonphoton mode splitting in a semiconductor quantum microcavity. Phys. Rev. Lett. 69, 3314 (1992).

19. Kasprzak, J., Richard, M., Kundermann, S., Baas, A., Jeambrun, P., Keeling, J. M. J., Marchetti, F. M., Szymańska, M. H., André, R., Staehli, J. L., Savona, V., Littlewood, P. B., Deveaud, B. \& Dang, Le Si Bose-Einstein condensation of exciton polaritons. Nature 443, 409-414 (2006).

20. Carusotto, I. \& Ciuti, C. Quantum fluids of light. Rev. Mod. Phys. 85, 299 (2013). 
21. Schneider, C., Winkler, K., Fraser, M.D., Kamp, M., Yamamoto, Y., Ostrovskaya, E. A. \& Höfling, S. Exciton-Polariton Trapping and Potential Landscape Engineering. Rep. Prog. Phys. 80, 016503 (2017).

22. St-Jean, P., Goblot, V., Galopin, E., Lemaître, A., Ozawa, T., Le Gratiet, L., Sagnes, I., Bloch, J. \& Amo, A. Lasing in topological edge states of a one-dimensional lattice. Nat. Photon. 11, 651656 (2017).

23. Peleg, O., Bartal, G., Freedman, B., Manela, O., Segev, M., and Christodoulides, D. N., Conical Diffraction and Gap Solitons in Honeycomb Photonic Lattices, Phys. Rev. Lett. 98, 103901 (2007).

24. Jacqmin, T. et al. Direct Observation of Dirac Cones and a Flatband in a Honeycomb Lattice for Polaritons. Phys. Rev. Lett. 112, 116402 (2014).

25. Milićević, M., Ozawa, T., Andreakou, P., Carusotto, I., Jacqmin, T., Galopin, E., Lemaître, A., Le Gratiet, L., Sagnes, I. \& Bloch, J. Edge states in polariton honeycomb lattices. 2D Materials 2, 034012 (2015).

26. Fujita, M., Wakabayashi, K., Nakada, K., and Kusakabe, K. Peculiar Localized State at Zigzag Graphite Edge. J. Phys. Soc. Jpn. 65, 1920 (1996).

27. Castro Neto, A. H., Guinea, F., Peres, N. M. R., Novoselov, K. S. and Geim, A. K. The electronic properties of graphene. Rev. Mod. Phys. 81, 109 (2009).

28. Yao, Y., Ye, F., Qi, X.-L., Zhang, S.-C., and Fang, Z. Spin-orbit gap of graphene: First-principles calculations. Phys. Rev. B 75, 041401(R) (2007).

29. Suchomel, H., Klembt, S., Harder, T., Klaas, M., Winkler, K., Emmerling, M., Höfling, S. \& Schneider, C. A plug and play platform for electrically pumped polariton simulators and topological lasers. arXiv:1803.08306 (2018).

30. Bahari, B., Ndao, A., Vallini, F., El Amili, A., Fainman, Y. \& Kanté, B. Nonreciprocal lasing in topological cavities of arbitrary geometries. Science 358, 636-640 (2017).

31. Bandres, M. A., Wittek, S., Harari, G., Parto, M., Ren, Jinhan, Segev, M., Christodoulides, D. N. \& Khajavikhan, M. Topological Insulator Laser Part II: Experiments. Science 359, 1231 (2018).

32. Lumer, Y., Plotnik, Y., Rechtsman, M.C., and Segev, M. Self-Localized States in Photonic Topological Insulators. Phys. Rev Lett. 111, 243905 (2013).

33. Kartashov, Y. V. \& Skryabin, D. V. Modulational instability and solitary waves in polariton topological insulators. Optica 3, 1228 (2016).

\section{Acknowledgements}

The authors thank R. Thomale for fruitful discussions. S.K. acknowledges the European Commission for the H2020 Marie Skłodowska-Curie Actions (MSCA) fellowship (Topopolis). S.K., S.H. and M.S. are grateful for financial support by the JMU-Technion seed money program. S.H. also acknowledges support by the EPSRC "Hybrid Polaritonics” Grant (EP/M025330/1). The Würzburg group acknowledges support by the ImPACT Program, Japan Science and Technology Agency and the ENB program (Tols 836315) of the State of Bavaria. T.C.H.L. and R. G. were supported by the Ministry of Education (Singapore) Grant No. 2017-T2-1-001. 


\section{Authors contributions}

S.K., M.S., C.S. and S.H. initiated the study and guided the work. S.K., T.H., K.W., M.E. and S.H. designed and fabricated the device. S.K. and T.H. performed optical measurements. S.K., T.H., O.A.E. and C.S. analyzed and interpreted the experimental data. O.A.E., R.G., T.C.H. L., M.B. and M.S. developed the

theory. S.K., T.H., O.A.E., T.C.H. L., C. S., M.S. and S. H. wrote the manuscript, with input from all coauthors.

\section{Additional information}

Competing financial interests: The authors declare no competing financial interests.

Correspondence and requests for materials should be addressed to Sebastian Klembt (sebastian.klembt@physik.uni-wuerzburg.de) and Sven Höfling (sven.hoefling@physik.uniwuerzburg.de) 
Figure 1 | Experimental scheme and theoretically calculated Bloch mode and dynamics calculations of topological polariton edge modes. (a) Schematic of the non-resonant laser excitation of left moving (yellow) and right moving (red) chiral topological polariton edge modes. (b, c) Trivial and topological band structures of the polariton honeycomb lattice for zero Zeeman splitting (b) and with Zeeman splitting $\Delta$ eff $=0.2 \mathrm{meV}$, induced by the external magnetic field (c). One-way topological edge modes are presented by red and blue lines within the topological gap. (d) Schematic and (e) calculated intensity profiles of the edge modes. (f, g) Calculated propagation dynamics of edge modes injected coherently (red circle) into the topological gap. (f) Left moving time-sequence (yellow) for negative Zeeman splitting $\Delta_{\mathrm{B}}=-0.8 \mathrm{meV}$ and (g) right moving propagation (red) for positive Zeeman splitting $\Delta_{\mathrm{B}}=+0.8 \mathrm{meV}$.

Figure 2 | Lattice device layout and geometry. (a-c) Scanning electron microscope images of the processed polariton honeycomb lattice. (a) Cleaved cross section of the microcavity prior to processing. (b) Tilted view of the half-etched honeycomb lattice for pillars with a diameter of $d=2.0 \mu \mathrm{m}$ and an overlap $v=a / d=0.85$. (c) Cleaved cross-section after etching, showing that only the top DBR has been etched. (d) Real-space honeycomb unit cell with two-element basis. (e) First Brillouin zone of the honeycomb lattice, featuring the six $K$ - and $K$ '-points. (f) Measured Fourier-space energy-resolved photoluminescence of the investigated lattice. The blue data points are fitted to the measured dispersions, agreeing well with a tightbinding model (red/yellow), accurately revealing the six Dirac cones at the $K$ - and $K$ '-points.

Figure 3 | Photoluminescence measurements of a polariton condensate in a topological edge mode. (a) Polariton dispersion along $K^{\prime} \rightarrow \Gamma \rightarrow K$ direction at $B=0 \mathrm{~T}$ compared with the calculated Bloch mode model (black dots). (b) Equivalent dispersions at $B=+5 \mathrm{~T}$, below and (c) above the threshold $P_{\mathrm{thr}}=1.8 \mathrm{~mW}$, where condensation into the $K, K^{\prime}$-points of the $S$-band is observed. Bloch mode calculations (red dots) show a distinct gap. (d) Real-space spectrum in $x$-direction along the straight white line in (g). The $x$-axis in Fig. 3d, e and g is the same. A trivial $S$-band condensate throughout the structure and a mode $(E=1.4678 \mathrm{eV})$ well separated from the bulk, located at the zigzag edge (dashed white line) are observed. (e) Mode tomography displaying a homogeneous trivial $S$-band condensate $\left(E_{S}=1.4673-1.4675 \mathrm{eV}\right)$ within the pump spot diameter of $40 \mu \mathrm{m}$. The inset shows a microscopy image of the structure. (f) Mode tomography of the topological edge mode $\left(E_{\text {edge }}=1.4678 \mathrm{eV}\right)$ at the corner position of the sample and $(\mathrm{g})$ at the same position as in (e). The mode is well located at the zigzag edge and clearly extends around the corner to the armchair configuration.

Figure 4 | Chirality and propagation of the condensate. Spectroscopic hyperspectral measurement of the full $S$-band condensate $(1.467-1.468 \mathrm{eV})$ at the $K, K^{\prime}$-points, including the energies linked to the topological edge modes for (a) $B=+5 \mathrm{~T}$ and (b) $B=-5 \mathrm{~T}$. The zigzag edge is oriented in $y$-direction. (c) Polariton intensity ratio between the $K^{\prime}$ - and $K$-points in $k_{\mathrm{y}}$-direction $\left(k_{\mathrm{x}} \simeq 0\right)$ as a function of the applied magnetic field. The dominant propagation direction is inverted (yellow/red arrows) when the direction of the magnetic field is reversed. For $B=0 \mathrm{~T}$ no dominant propagation direction is observed. The error bars originate from image distortions, inhomogeneities of the excitation, and uncertainties during data processing, and are estimated at 5\%. 


\section{Methods Section}

\section{Basic sample characterization}

Honeycomb lattices of coupled micropillars were etched into a planar semiconductor Fabry-Pérot microcavity, grown by molecular beam epitaxy. The cavity consists of a GaAs $\lambda$-cavity equipped with three $16 \mathrm{~nm}$ wide $\mathrm{In}_{0.04} \mathrm{Ga}_{0.96}$ As quantum wells sandwiched between two distributed Bragg reflectors with 30 (35.5) $\mathrm{Al}_{0.10} \mathrm{Ga}_{0.90} \mathrm{As} / \mathrm{AlAs}$ top (bottom) mirror pairs. The quantum wells were placed at the maximum of the electromagnetic field, resulting in a strong exciton-photon coupling with a Rabi splitting of $4.3 \mathrm{meV}$ (see extended data Fig. E1). The set of quantum wells emit with an exciton linewidth of $\gamma_{\mathrm{X}}=1.2 \mathrm{meV}$ (full width at half maximum), measured with the top mirror etched away. The quality factor of the cavity was determined experimentally by measuring the mode linewidth of a single large pillar at a negative (photonic) detuning $\delta \sim-11.5 \mathrm{meV}$ to be $\sim 13.000\left(\gamma_{\mathrm{C}}=0.11 \mathrm{meV}\right)$. The overall layer thicknesses decrease radially towards the outside of the wafer, effecting mainly the photonic mode, allowing to choose a certain exciton to photon detuning. By increasing the asymmetry between the cavity mode and the stopband center, the TE-TM splitting of the cavity mode is increased, mimicking an effective spin-orbit interaction [34]. However, the further the cavity mode is moved towards the edge of the stopband, the lower the reflectivity becomes, implying a lower Q-factor and larger polariton linewidth. Based on simulations and experience from previously grown samples, a DBR (cavity) asymmetry factor of $1.03(0.91)$ - $1.00(1.00)$ would be the symmetric case - was chosen for the microcavities presented in this work, yielding a TE-TM splitting of approximately $600 \mu \mathrm{eV}$ at $k_{\|}=2.0 \mu \mathrm{m}^{-1}$ (see extended data Fig. E2). Both the Indium content in the InGaAs quantum well and the quantum well thickness were optimized with regards to the smallest linewidth and largest Zeeman splitting. 4\% Indium and $16 \mathrm{~nm}$ thickness yield a Zeeman splitting $\Delta E_{\mathrm{Z}}=540 \mu \mathrm{eV}$ at $B=5 \mathrm{~T}$ (see extended data Fig. E2). After sample growth, a honeycomb lattice of approximately $120 \times 120 \mu \mathrm{m}^{2}$ with a pillar diameter of $\mathrm{d}=2.0 \mu \mathrm{m}$ and an overlap $v=a / d=0.85$ was defined using an electron beam lithography process and 
subsequent wet etching. Extended data Fig. E3 shows a microscope image of an intended defect in the form of a site missing in a zigzag edge of the honeycomb lattice (indicated by the red arrow).

\section{PL experiment description - Methods}

Non-resonant photoluminescence experiments with and without an applied magnetic field were carried out using a linearly polarized, pulsed titanium-sapphire laser with a repetition rate of $82 \mathrm{MHz}$ and a pulse length of approximately 2 ps. The wavelength of the laser was set to be $\lambda_{\mathrm{L}}=792 \mathrm{~nm}$, coinciding with the first high-energy stopband minimum of the microcavity structure.

The emission was collected using a microscope objective $(20 \mathrm{x}, \mathrm{NA}=0.4)$ and imaged at the entrance slit of a Czerny-Turner spectrometer, equipped with a charge-coupled device (CCD) camera with a resolution of $\sim 20 \mu \mathrm{eV}$. A motorized imaging lens allows for automated hyperspectral images $\left(\left(k_{\mathrm{x}}, k_{\mathrm{y}}\right) \mathrm{vs}\right.$. $\left.E\right)$ and mode tomographies $((x, y)$ vs. $E)$.

The sample was mounted in a liquid Helium flow cryostat (Oxford, SpectraMag), operating at a temperature of $T=4 \mathrm{~K}$. Using superconducting coils, a magnetic field $B=-5 \mathrm{~T}$ to $+5 \mathrm{~T}$ can be applied in Faraday geometry.

\section{Bloch mode calculations for polarion honeycomb lattices}

A widely accepted model describes the dynamics of excitons with spin-up $\left(\psi^{+}\right)$and spin-down

$\left(\psi^{-}\right)$coupled to cavity photons carrying the right $\left(E^{+}\right)$and the left $\left(E^{-}\right)$circular polarizations, respectively [s1], and is governed by

$$
\begin{gathered}
i \hbar \partial_{t} E^{ \pm}=\left(-\frac{\hbar^{2}}{2 m_{C}} \nabla_{\perp}^{2}+V(\mathbf{r})+\omega_{C}-i \gamma\right) E^{ \pm}+\beta\left(\partial_{x} \mp i \partial_{y}\right)^{2} E^{\mp}+\hbar \Omega_{R} \psi^{ \pm}+E_{p}^{ \pm} e^{-i \omega_{p} t} \\
i \hbar \partial_{t} \psi^{ \pm}=\left(\omega_{E}-i \gamma \pm \frac{\Delta_{B}}{2}\right) \psi^{ \pm}+\hbar \Omega_{R} E^{ \pm}
\end{gathered}
$$

Here, the normalization is such that $\left|\mathrm{E}^{ \pm}\right|^{2}$ and $\left|\psi^{ \pm}\right|^{2}$ are the number of particles per unit area. The quantities $\omega_{\mathrm{C}}$ and $\omega_{\mathrm{E}}$ represent the energies of bare photons and excitons, respectively. In the present configuration the photon-exciton detuning is negative $\delta=\left(\omega_{\mathrm{C}}-\omega_{\mathrm{E}}\right)=-6 \mathrm{meV}$. The photon-exciton coupling strength is 
given by the parameter $\hbar \Omega_{\mathrm{R}}$, which defines the Rabi splitting as $2 \hbar \Omega_{\mathrm{R}}=4.5 \mathrm{meV}$. Here, $m_{\mathrm{C}}=32.3 * 10^{-}$ ${ }^{6} m_{\mathrm{e}}$ is the effective photon mass in the planar region and $m_{\mathrm{e}}$ is the free electron mass. The effective mass of excitons is $m_{\mathrm{E}} \simeq 10^{5} \mathrm{~m}_{\mathrm{C}}$. An external photonic potential $V(\boldsymbol{r})$ is defined within the unit cell of the honeycomb structure, constructed of circular mesas (micropillars). We assume that the potential is $V(\boldsymbol{r})=30 \mathrm{meV}$ outside the mesas and zero otherwise. In what follows, we assume that the intensity of polaritons is weak enough and thus neglect nonlinear interactions between them. The TE-TM splitting of the cavity modes gives rise to the linear coupling between right- and left- circular polarizations and is denoted by $\beta$ [35]. We account for the magnetic field via Zeeman splitting $\Delta_{\mathrm{B}}$ of the excitonic states in the quantum wells.

Equations (1.1) and (1.2) allow for accurate simulation of the propagation of the chiral modes coherently injected into the system by means of the external coherent radiation $\left(E_{p}^{ \pm}\right)$, with an appropriately chosen frequency $\left(\omega_{\mathrm{p}}\right)$ within the topological gap. First, we calculate the energy-momentum band structure of the honeycomb lattices, using a full description of the Bloch modes taking into account all relevant system parameters. For this aim, we solve the following eigenvalue problem for the energy $\hbar \mu\left(\boldsymbol{k}_{\mathrm{b}}\right)$ of the Bloch mode with the Bloch vector $\boldsymbol{k}_{\mathrm{b}}=\left\{k_{\mathrm{bx}}, k_{\mathrm{by}}\right\}$

$$
\hbar \mu\left(\boldsymbol{k}_{b}\right)\left\{\begin{array}{l}
\boldsymbol{p}_{b}^{+}\left(r, k_{b}\right) \\
\boldsymbol{p}_{b}^{-}\left(r, k_{b}\right)
\end{array}\right\}=\left[\begin{array}{cc}
\hat{\mathrm{L}} & \hat{\mathrm{C}}^{+} \\
\hat{\mathrm{C}}^{-} & \hat{\mathrm{L}}
\end{array}\right]\left\{\begin{array}{l}
\boldsymbol{p}_{b}^{+}\left(r, k_{b}\right) \\
\boldsymbol{p}_{b}^{-}\left(r, k_{b}\right)
\end{array}\right\}
$$

The circularly polarized polaritonic wave-functions are $\boldsymbol{p}_{b}^{ \pm}\left(\boldsymbol{r}, \boldsymbol{k}_{\boldsymbol{b}}\right)=\left\{c_{b}^{ \pm}\left(\boldsymbol{r}, \boldsymbol{k}_{\boldsymbol{b}}\right), x_{b}^{ \pm}\left(\boldsymbol{r}, \boldsymbol{k}_{\boldsymbol{b}}\right)\right\}$, where the functions $c_{b}^{ \pm}\left(\boldsymbol{r}, \boldsymbol{k}_{\boldsymbol{b}}\right)$ and $x_{b}^{ \pm}\left(\boldsymbol{r}, \boldsymbol{k}_{\boldsymbol{b}}\right)$ describe the amplitude distributions of the photonic and excitonic component of the Bloch modes in real space, defined in the plane of the microcavity $\boldsymbol{r}=\{x, y\}$. The diagonal of the matrix in eqn. (2) describes the single-particle coupled states of excitons and photons and is given by the expression

$$
\hat{L}=\left(\begin{array}{cc}
\omega_{C}+V(\boldsymbol{r})-\frac{\hbar^{2}}{2 m_{C}}\left(\vec{\nabla}_{\perp}+i \overrightarrow{k_{b}}\right)^{2} & \hbar \Omega_{R} \\
\hbar \Omega_{R} & \omega_{E}-\frac{\hbar^{2}}{2 m_{E}}\left(\vec{\nabla}_{\perp}+i \overrightarrow{k_{b}}\right)^{2}
\end{array}\right)
$$


The coupling between both polarization components is given by the off-diagonal matrices $\hat{\mathrm{C}}^{ \pm}$

$$
\hat{C}^{ \pm}=\left(\begin{array}{cc}
\beta\left(\left(\vec{\nabla}_{\perp}+i \overrightarrow{k_{b}}\right)\left(e_{\mathbf{x}} \mp i e_{\mathbf{y}}\right)\right)^{2} & 0 \\
0 & \pm \frac{\Delta_{B}}{2}
\end{array}\right),
$$

which includes both the TE-TM splitting of the photonic modes and the Zeeman splitting of the excitons due to the applied magnetic field. The unit vectors $\boldsymbol{e}_{x, y}$ shows the directions of cartesian coordinates $(x, y)$ in the plane of the microcavity. To reduce drastically computational efforts, it is convenient to solve the eigenvalue problem in polaritonic basis after diagonalization of the matrix $\hat{L}$, describing the coupling between photons and excitons. In this case, the effective parameter for the TE-TM splitting $\beta_{\text {eff }} \sim \beta|C|^{2}$ and the Zeeman splitting $\Delta_{\text {eff }} \sim \Delta_{\mathrm{B}}|X|^{2}$ are scaled with the photonic and excitonic components of the polaritons, respectively (where $|C|^{2}$ and $|X|^{2}$ are the Hopfield coefficients, calculated for the respective Bloch mode.

Physically, the excitation occurs by coherent illumination with linearly polarized light at the frequency within the topological gap. The seeding pulse duration is about 40 ps. Other parameters: $\Delta_{\mathrm{B}}= \pm 0.8 \mathrm{meV}$, $\beta=0.20 \mathrm{meV} \mu \mathrm{m}^{2}, \beta_{\text {eff }}=0.15 \mathrm{meV} \mu \mathrm{m}^{2}, \gamma=0.01 \mathrm{meV}$, polariton lifetime $\tau=35 \mathrm{ps}$, pillar diameter $d=2.0 \mu \mathrm{m}$, center-to-center separations $a=2 \mu \mathrm{m}$.

\section{Polariton chiral edge mode propagating around a corner and defect.}

Using the Bloch mode calculations described above, we now excite the system resonantly and calculate its evolution in time for a Zeeman splitting of $\Delta_{\mathrm{B}}=+0.8 \mathrm{meV}$. The polaritons propagate along the edge and around the $90^{\circ}$ corner as expected for a topological edge mode (see Fig. E4). In the same way we find that the edge mode avoids an artificial defect in form of a site missing in a zigzag chain (see Fig. E5). 


\section{Tight-binding model}

A tight-binding model describing the artificial graphene band structure is given in eqns. (5) and (6). Here, $t$ is the nearest neighbor, $t^{\star}$ the next-nearest neighbor interaction and $\boldsymbol{k}_{\|}$the measured in-plane lattice vector being linked to the measured polariton emission angle $\theta$ by $k_{\|}=(\omega / c) \sin (\theta)$.

$$
\begin{gathered}
E_{h c}\left(\boldsymbol{k}_{\|}\right)=E_{0} \pm t \sqrt{3+f\left(\boldsymbol{k}_{\|}\right)}-t^{\prime} f\left(\boldsymbol{k}_{\|}\right) \\
f\left(\boldsymbol{k}_{\|}\right)=2 \cos \left(\sqrt{3} k_{y} a\right)+4 \cos \left(\frac{\sqrt{3}}{2} k_{y} a\right) \cos \left(\frac{3}{2} k_{x} a\right)
\end{gathered}
$$

The resulting band structure of this model is presented as a fit to the data in Fig. 2 (f) of the main text in red and yellow.

\section{Gap measurement}

When an external magnetic field is applied to this lattice, a band gap is predicted to open at the Dirac points. We make use of a $\lambda / 4$ polarisation series to detect this gap. In Extended Data Fig. E6, exemplary images of the $\lambda / 4$-series at external magnetic fields of $B=0 \mathrm{~T}$ and $5 \mathrm{~T}$ are presented. The energetic position of the Dirac point was evaluated by fitting a Lorentzian peak profile to the line spectrum through the Dirac point at $K^{\prime}$. The peak positions were plotted against the angle of the $\lambda / 4$-waveplate. Assuming that the position of the peak corresponds linearly to its polarisation, the resulting graph can be fitted using equation

$$
I(\phi)=\frac{1}{2}\left(S_{0}+S_{1} \cos ^{2}(2 \phi)+S_{2} \sin (2 \phi) \cos (2 \phi)+S_{3} \sin (2 \phi)\right)
$$

normally used to fit the peak intensity in a $\lambda / 4$-series with a constant peak position. At $B=+5 \mathrm{~T}$ a band gap of $E_{\mathrm{g}}=(108 \pm 32) \mu \mathrm{eV}$ was evaluated. The peak movement with no external magnetic field applied 
can be attributed to imperfections of the $\lambda / 4$-waveplate and was used as an uncertainty of the band gap that opens when an external magnetic field is applied.

\section{Input/Output characteristics and linewidth}

By increasing the power of the non-resonant pump laser, measuring the intensity and linewidth we determine the threshold characteristic of the polaritons as shown in extended data Fig. E7. At a typical threshold of $P_{\text {thr }} \approx 1.8 \mathrm{~mW}$ a distinct non-linear increase in intensity as well as a sudden decrease in linewidth can be observed. The mode accociated with the gap mode becomes visible around $P=P_{\text {thr }}$.

\section{Ginzburg-Landau calculations of polariton condensation into a topological edge mode}

In theoretical proposals such as [15-17]; no particular form of excitation was considered explicitly. It is clear from section (S1) that a resonant excitation is able to directly populate the various modes. For the case of non-resonant excitation, further theoretical work is needed to access the physics of polariton condensation into a chiral topological egde mode, described in Fig. 3 of the main text.

Here we use the driven-dissipative Ginzburg-Landau model, frequently used to describe the spatial form of polariton condensates:

$i \hbar \frac{\partial}{\partial t}\left(\begin{array}{l}\psi_{+} \\ \psi_{-}\end{array}\right)=\left(\begin{array}{cc}\hat{E}_{0}+V(\boldsymbol{r})+i W(\boldsymbol{r})+\frac{\Delta_{e f f}}{2}-i\left|\psi_{+}\right|^{2} & \beta_{e f f}\left(\hat{k}_{x}-i \hat{k}_{y}\right)^{2} \\ \beta_{e f f}\left(\hat{k}_{x}+i \hat{k}_{y}\right)^{2} & \hat{E}_{0}+V(\boldsymbol{r})+i W(\boldsymbol{r})-\frac{\Delta_{e f f}}{2}-i\left|\psi_{-}\right|^{2}\end{array}\right)\left(\begin{array}{l}\psi_{+} \\ \psi_{-}\end{array}\right)$ 
Here $\psi_{+}$and $\psi_{-}$denote the two spin components of the two-dimensional polariton wavefunction. The operator $\widehat{E}_{0}$ corresponds to the bare kinetic energy of polaritons, modelled with an effective mass $m$. The potential $V(\boldsymbol{r})$ accounts for the honeycomb lattice structure and $\Delta_{\text {eff }}$ is the Zeeman splitting caused by the applied magnetic field. The two spin components are coupled by the off-diagonal spin-orbit coupling term, physically corresponding to a TE-TM splitting with strength $\beta_{\text {eff. }}$.

The linear gain and loss in the system is described by the term $W(\boldsymbol{r})=P(\boldsymbol{r})-\Gamma(\boldsymbol{r})$, where $P(\boldsymbol{r})$ represents the spatially dependent non-resonant pumping and $\Gamma$ is a spatially dependent dissipation rate. We assume that the loss is higher outside the micropillar regions. For the system to form a steady state it is important to consider also nonlinear loss terms. For simplicity, we neglect the effect of polariton-polariton interactions, which is valid provided we operate not too far above the condensation threshold.

Before solving the full nonlinear problem, it is instructive to consider the spectrum of linearized modes, obtained by neglecting the nonlinear term. Considering a strip geometry, where the potential is periodic in the $x$-direction and bounded in the orthogonal $y$-direction, the Bloch theory can be used to obtain the complex bandstructure of the system eigenmodes with respect to a wavevector $k_{\mathrm{x}}$. The result is shown in Extended Data Fig. E8a. For parameters comparable with the experiment, we obtain a topological bandgap bridged by chiral edge states. A slight asymmetry in the edge states occurs due to the pumping $P(\boldsymbol{r})$, which is taken periodic in the $x$-direction (to maintain validity of the Bloch theory) but preferentially localized near the bottom edge of the strip.

Extended Data Fig. E8b shows the imaginary parts of the same eigenmodes. Remarkably, the chiral edge state is found to have the largest imaginary component, meaning that it has a larger gain than other states and would be preferentially selected during polariton condensation. However, it should be noted that in principle there are an infinite number of energy states obtainable with the present theory and it is in practice necessary to introduce an energy cutoff to solve the Bloch Hamiltonian. 
To rule out the potential population of higher energy states, we rely on solution of the full nonlinear problem, propagating eqn. (7) in time to a steady state. Here we indeed find condensation in a chiral edge state, as illustrated with the color scale in Extended Data Fig. E8a. A slight shift from the results of the linear bandstructure calculation occurs due to the nonlinear term. Extended Data Fig. E8c shows the obtained wavefunction in real space, which is indeed localized at the edge excited by the pumping, perfectly agreeing with the experimental findings. In addition to explaining our experimental observation of chiral current under non-resonant pump, these theoretical results predict a new phenomenon of polariton lasing in a topological edge state: a topological polariton laser [30,31]. However, we point out that a strict comparison between a topological laser and a non-topological laser (as in [31]) cannot be easily presented, since the topological gap cannot be closed, without changing a wide range of other system parameters.

\section{Real-space mode tomographies at $B=0 \mathrm{~T}$ and $B=-5 \mathrm{~T}$}

For the sake of completeness, we perform the same mode tomographies, displayed in the main text in Fig. 3 at $B=+5 \mathrm{~T}$ at $B=0 \mathrm{~T}$ and $B=-5 \mathrm{~T}$. The results are displayed in Extended Data Fig. E9 (a-d, $B=0 \mathrm{~T}$;

e-h, $B=-5 \mathrm{~T}$ ). While for the $B=0 \mathrm{~T}$ case, no distinct edge mode is observed, the behavior for $B=-5 \mathrm{~T}$ is qualitatively similar to $B=+5 \mathrm{~T}$, as expected.

\section{Extended References}

34. Sala, V. G., Solnyshkov, D. D., Carusotto, I., Jacqmin, T., Lemaître, A., Terças, H., Nalitov, A., Abbarchi, M., Galopin, E., Sagnes, I., Bloch, J., Malpuech, G. \& Amo, A. Spin-Orbit Coupling for Photons and Polaritons in Microstructures. Phys. Rev. X 5, 011034 (2015).

35. Deveaud, B. (Ed.) The Physics of Semiconductor Microcavities (2007) 
Data availability. The data that support the findings of this study are available from the corresponding authors on reasonable request.

\section{Extended Data Figure Legends}

Extended Data Figure E1 | White light reflectivity measurements as a function of the detuning. Reflectivity measurements as a function of the detuning. A distinct anticrossing behavior, with a Rabi splitting of $2 \hbar \Omega_{\mathrm{R}}=4.3 \mathrm{meV}$ can be observed. The measurements were performed on a sample piece with approximately 15 mirror pairs removed from the top DBR in order to increase the signal quality. Inset: Fitted peak positions vs. detuning.

Extended Data Figure E2 | Zeeman splitting and TE-TM splitting for a III-V microcavity hosting Ino.04Ga0.96As quantum wells. (left) Zeeman-splitting with regard to the magnetic field including second order polynomial fits as a guide to the eye. Sample A is the one used in this experiment. Inset: Exemplary data of central emission energies for a $\lambda / 4$-series with a sine fit. (right) Experimentally determined TE-TM-splittings at various detunings $\Delta \mathrm{E}$ for sample A including fits with modified photonic Hopfield coefficients (red).

Extended Data Figure E3| Microscopy image of a zigzag edge polariton honeycomb lattice with an intended defect. Image of the honeycomb lattice with $d=2.0 \mu \mathrm{m}$ and $v=0.85$, with an intended defect in the zigzag chain, selected for experiments in an external magnetic field.

Extended Data Figure E4 | Polariton chiral edge mode propagating around a corner. (a-c) Propagation dynamics of edge modes injected coherently into the topological gap and calculated within the model 1 . Shown is the right moving propagation for the positive value splitting $\Delta \mathrm{B}=+0.8 \mathrm{meV}\left(\left|\Delta_{\mathrm{eff}}\right|=0.2 \mathrm{meV}\right)$ and $\beta=0.20 \mathrm{meV} \mu \mathrm{m}^{2}\left(\beta_{\mathrm{eff}}=0.15 \mathrm{meV}_{\mu} \mathrm{m}^{2}\right)$. A linearly-polarized narrow coherent seeding beam injects both polarization components into the mesa marked by the red circle. At $t \approx 100$ ps the mode propagates around the corner from the zigzag edge into the armchair one.

Extended Data Figure E5 | Polariton chiral edge mode propagating and avoiding a defect. (a-c) Propagation dynamics of edge modes injected coherently into the topological gap and calculated within the model 1 . Shown is the right moving propagation for the positive value splitting $\Delta_{\mathrm{B}}=+0.8 \mathrm{meV}\left(\left|\Delta_{\mathrm{eff}}\right|=0.2 \mathrm{meV}\right)$ and $\beta=0.20 \mathrm{meV} \mu \mathrm{m}^{2}\left(\beta_{\mathrm{eff}}=0.15 \mathrm{meV}^{2}\right)$. A linearly-polarized narrow coherent seeding beam injects both polarization components into the mesa. At $t \approx 85$ ps the mode propagates around the defect in the zigzag chain, marked by the red circle. 
Extended Data Figure E6| Topological gap measurement. $\lambda / 4$-plate measurement at $B=0 \mathrm{~T}$ (blue) and $B=+5 \mathrm{~T}$ (red) at the $K$-point yielding a band gap of $E_{\mathrm{g}}=(108 \pm 32) \mu \mathrm{eV}$.

\section{Extended Data Figure E7 | Input/Output characteristics and linewidth behavior as a function of pump power. Below} threshold the gap and bulk mode cannot be distinguised. At a typical threshold $P_{\text {th }} \approx 1.8 \mathrm{~mW}$ a distinct non-linear increase in intensity as well as a sudden decrease in linewidth can be observed. Here, the populated gap modes show similar behavior to the bulk mode.

\section{Extended Data Figure E8 | Driven-dissipative Gross-Pitaevskii calculation of polariton condensation into topological edge} mode. (a) Bandstructure of polaritons in a honeycomb lattice. The dotted curves represent the dispersion of the linear eigenmodes of a strip, color coded to represent the localization on the bottom edge (red), upper edge (green) and in the bulk (blue). The shaded region represents the energy and momentum of the polariton steady state from solving the driven-dissipative Gross-Pitaevskii equation. (b) Imaginary components of the linear eigenmodes. The largest imaginary part corresponds to an edge state (the color coding is the same as in (a)), suggesting that the edge state is most likely to be populated with increasing pumping. (c) Edge state obtained from solution of the driven-dissipative Gross-Pitaevskii equation. Parameters: $\Delta$ eff $=0.3 \mathrm{meV}$, $\beta_{\text {eff }}=0.2 \mathrm{meV} \mu \mathrm{m}^{2}$. The effective mass, $m$, was taken as $1.3^{*} 10^{-4}$ of the free electron mass; the potential of depth $0.5 \mathrm{meV}$ was constructed from a honeycomb lattice of cylinders of radius $1 \mu \mathrm{m}$ and center-center separation $1.7 \mu \mathrm{m}$; the pump spot was taken as a Gaussian centered on the strip edge with extent $7.5 \mu \mathrm{m}$ in the $y$-direction. A spatially uniform decay rate of $0.2 \mathrm{meV}$ was supplemented with a $1.7 \mathrm{meV}$ decay in the region outside the cylinders.

Extended Data Figure E9 | Real-space mode tomographies of a polariton condensate at $B=0 \mathrm{~T}$ and $B=-5 \mathrm{~T}$. (a) $-(\mathrm{d})$ Measurements at $\boldsymbol{B}=\mathbf{0}$ T. (a) Real-space spectrum in $x$-direction perpendicular to the zigzag edge along the straight white line in (d). The real-space $x$-axis is consistent between Extended data Figs. E9 a, b and d. The dashed white line marks the physical edge of the lattice. Only a trivial $S$-band condensate can be observed throughout the structure. (b) Mode tomography displaying the topologically trivial $S$-band condensate at $E_{\mathrm{S}}=1.4673-1.4675 \mathrm{eV}$. A relatively homogeneous condensate within the pump spot diameter of $40 \mu \mathrm{m}$ is observed. The inset shows a microscopy image of the structure. (c) Mode tomography of the energy $E_{\text {edge }}=1.4678 \mathrm{eV}$ for comparison at the corner position and at the edge (d) of the sample. Without magnetic field, no localized edge mode can be observed. (e)-(h) Measurements at $\boldsymbol{B}=-\mathbf{5} \mathbf{T}$ (fully analogous to Fig. $3 \mathrm{~d}$-g in the main text). (e) Real-space spectrum in $x$-direction perpendicular to the zigzag edge along the straight white line in (h). The real-space $x$-axis is consistent between Extended data Figs. E9 e, f and h. The dashed white line marks the physical edge of the lattice. A trivial $S$-band condensate can be observed throughout the structure. At $E=1.4678 \mathrm{eV}$ again we observe the appearance of a localized mode, well separated from the bulk and located at the zigzag edge. (f) Mode tomography displaying the topologically trivial $S$-band condensate at $E_{\mathrm{S}}=1.4673-1.4675 \mathrm{eV}$. A relatively homogeneous condensate within the pump spot diameter of $40 \mu \mathrm{m}$ is observed. The inset shows a microscopy image of the structure. (g) Mode tomography of the topological edge mode at $E_{\text {edge }}=1.4678 \mathrm{eV}$ at the corner position and at the edge $(\mathbf{h})$ of the sample, showing clearly that the mode extends around the corner from the zigzag to the armchair configuration and avoids the intentional defect, both without bulk scattering. 